\title{
Pheochromocytoma of the pancreas: A report of three cases and a literature review
}

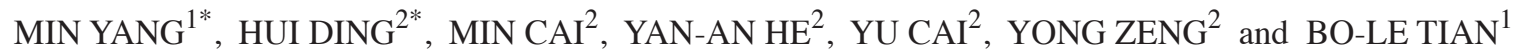 \\ ${ }^{1}$ Department of Pancreatic Surgery, West China Hospital of Sichuan University, Chengdu, Sichuan 610041; \\ ${ }^{2}$ Department of Hepatobiliary Surgery, People's Hospital of Jiangyou, Mianyang, Sichuan 621700, P.R. China
}

Received December 14, 2014; Accepted December 11, 2015

DOI: 10.3892/ol.2016.4721

\begin{abstract}
Pheochromocytoma is primarily derived from the adrenal medulla. The majority of extra-adrenal pheochromocytoma cases occur in the superior para-aortic region and para-adrenal area. However, pheochromocytoma originating from the pancreas is rare. The present study reports the cases of three patients who had no history of hypertension but were post-operatively diagnosed with pheochromocytoma located in the pancreas. Of the three patients, two were admitted to hospital due to abdominal pain, and imaging examinations revealed a soft-tissue lesion in the head of pancreas. Local resection of the pancreatic tumor was successfully performed and a diagnosis of pheochromocytoma derived from the pancreas was subsequently made by pathologists. The third patient was admitted to hospital for surgical treatment due to the identification of a continuously growing lesion in the tail of pancreas during physical examinations. Distal resection of the pancreas was stopped during surgery when the patient's blood pressure and heart rate suddenly increased to $180 / 110 \mathrm{mmHg}$ and 140 beats/min, respectively. Due to a marked rise in noradrenaline and adrenaline levels in the blood subsequent to surgery, the patient was diagnosed with pancreatic pheochromocytoma. The present study additionally reviewed the associated literature concerning pheochromocytoma in order to improve the understanding of this rare clinical phenomenon. The aim of the present study is to highlight to surgeons that although patients may not present with typical clinical manifestations due to the non-functional status of the tumor, undiagnosed pheochromocytoma of the pancreas should be considered when surgeons observe an unexpected hypertensive crisis during pancreatic tumor surgery.
\end{abstract}

Correspondence to: Mr. Hui Ding, Department of Hepatobiliary Surgery, People's Hospital of Jiangyou, 346 Jinlun Middle Road, Mianyang, Sichuan 621700, P.R. China

E-mail: dinghui3787@163.com

*Contributed equally

Key words: pancreas, pheochromocytoma, diagnosis, surgery

\section{Introduction}

Pheochromocytoma is a catecholamine-secreting tumor that originates from chromaffin cells. The majority of these tumors arise in the adrenal medulla (1), and when they occur outside the adrenal gland they are known as an extra-adrenal pheochromocytoma or a paraganglioma (2). Pheochromocytoma is primarily sporadic, but may additionally be associated with multiple endocrine neoplasia syndrome (3) and mutations of mitochondrial complex 2 succinate dehydrogenase enzymes (4).

Extra-adrenal pheochromocytoma has been previously reported to account for $\sim 10 \%$ of all pheochromocytoma cases during adulthood and for $30-40 \%$ of cases in children (5). These tumors may occur in the superior para-aortic region, the para-adrenal area and the inferior para-aortic region, as well as the organ of Zuckerkandl and the urinary bladder (6-8). Pancreatic localization of extra-adrenal pheochromocytoma is rare, and to the best of our knowledge, only one case has been previously reported in the Chinese literature (9). In the present study, the cases of three patients (Table I) with pheochromocytoma involving the pancreas are presented. To the best of our knowledge, the present report is the first to present three definite and comprehensive cases of this rare clinical phenomenon. In addition, the relevant literature is reviewed, with the aim of improving the understanding of pheochromocytoma, with an emphasis on sharing patient experiences of diagnosis and surgical treatment.

\section{Case report}

Case 1. A 55-year-old female was admitted to the People's Hospital of Jiangyou (Mianyang, China) in February 2013 with the symptom of recurrent abdominal pain over a period of $>3$ years. On admittance, the patient did not exhibit any weight loss, changes in appetite or bowel habits, jaundice or non-specific symptoms associated with hypertension, including palpitations, sweating, headache, dizziness or fainting. The patient's physical examination and pre-operative routine examinations did not identify any significant abnormalities. Serum tumor markers for cancer antigen 19-9, cancer antigen 125 and carcinoembryonic antigen (CEA) were additionally observed to be within the normal ranges. The patient underwent ultrasound (US) and computed tomography (CT; LightSpeed VCT CT 
Table I. Clinical features of three patients with pancreatic pheochromocytoma.

\begin{tabular}{lccc}
\hline Variable & Case 1 & Case 2 & Case 3 \\
\hline Gender & Female & Male & Female \\
Age, years & 55 & 44 & 30 \\
Hypertension & No & No & No \\
Family history & No & No & No \\
Chief complaint & Abdominal pain & Abdominal pain & Neoplasm detected on \\
& & & routine physical examination \\
Tumor location & Uncinate process & Uncinate process & Pancreatic tail \\
Tumor size, cm & 2.5 & 3.2 & 6 \\
Surgery & Local resection & Local resection & Laparotomy \\
Complications & No & Pancreatic fistula & Mortality \\
Patient outcome & Alive & Alive & Succumbed \\
\hline
\end{tabular}
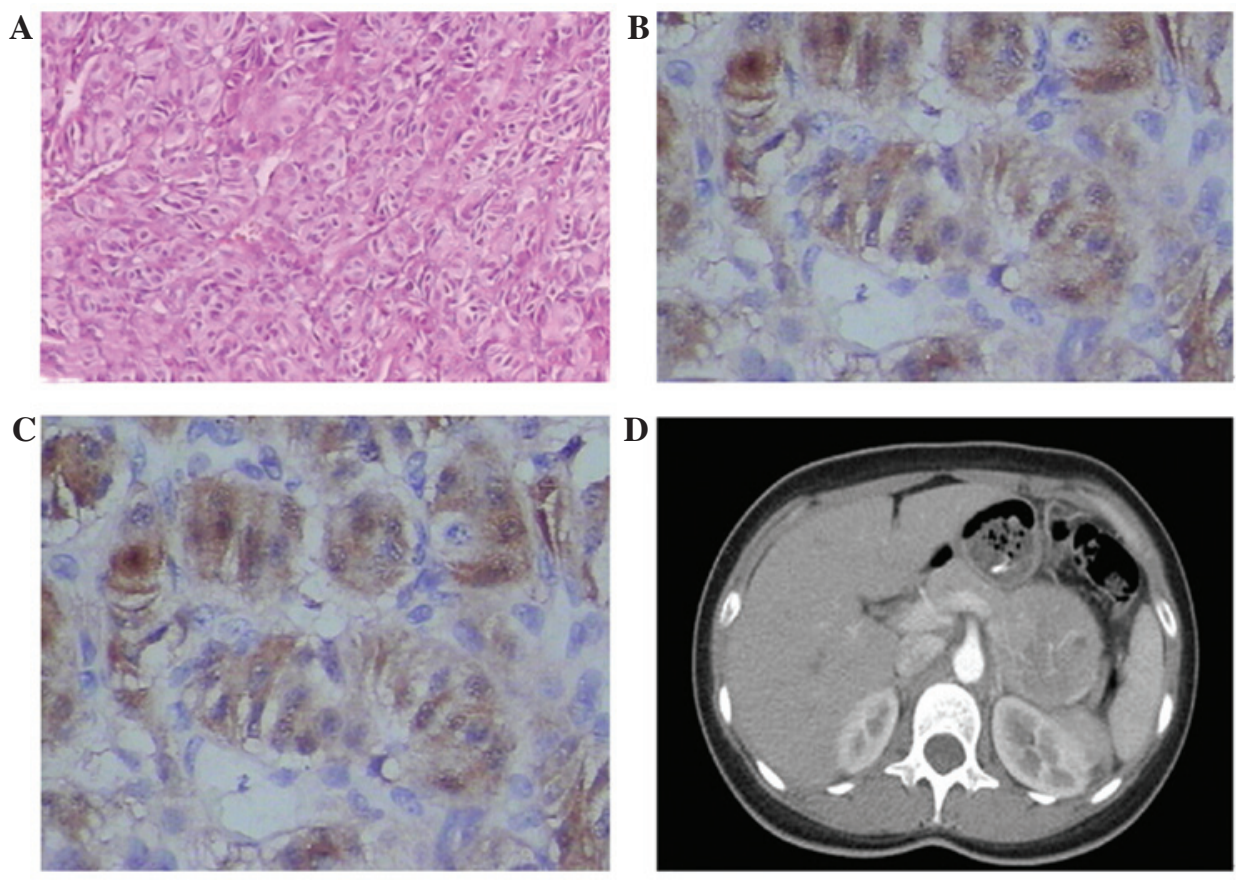

Figure 1. (A) Case 1: Hematoxylin and eosin staining of the resected tumor tissue showed a neuroendocrine tumor formed by round nests of densely packed cells, with palisading tumor cells (magnification, $\mathrm{x} 400$ ). (B) Case 1: Immunohistochemical staining revealed that the tumor tissue was positive for chromogranin A. (C) Case 2: Immunohistochemical staining revealed that the tumor tissue was positive for synaptophysin. (D) Case 3: Abdominal contrast-enhanced computed tomography scan of a circular neoplasm situated in the tail of the pancreas.

system; GE Healthcare Life Sciences, Chalfont, UK), which revealed a mass in the uncinate process of the pancreas, with a diameter of $\sim 2.5 \mathrm{~cm}$. Due to suspicions of a pancreatic malignant tumor, an exploratory laparotomy was performed, which confirmed the location of the mass in the uncinate process of the pancreas, with a relatively clear association with the surrounding tissues. A local resection of the pancreatic tumor was subsequently successfully performed. Resected tissue was formalin (Beijing Noble Ryder Technology Co., Ltd., Beijing, China)-fixed, paraffin (Beijing Noble Ryder Technology Co., Ltd.)-embedded and cut into 4- $\mu \mathrm{m}$ sections. For histological examination, the tissue sections were stained with hematoxylin and eosin (Haibiao Technology, Co., Ltd., Xiamen, China) and observed under a microscope (CX31; Olympus Coropration,
Tokyo, Japan). Pathologists were able to histologically diagnose the tumor as pancreatic paraganglioma (extra-adrenal pheochromocytoma originating from the pancreas). The tumor cells were polygonal, had abundant cytoplasm and contained basophilic or amphotropic particles The nuclei were round or irregular, and the tumor cells were arranged in cords or nests. According to the introperative and histological findings, the tumor was 2.5 and $3 \mathrm{~cm}$ in diameter, respectively. There was no evidence of distant metastasis, and no direct tissue or arterial or venous invasion. For immunohistochemical analysis, tissue sections were incubated with monoclonal mouse antihuman chromogranin A (CgA; cat. no. ZA0507; 1:50; Beijing Zhongshan Golden Bridge Biotechnology Co., Ltd., Beijing, China), monoclonal mouse anti-human synaptophysin (Syn; 
cat. no. M077629; 1:100; Dako, Glostrup, Denmark), S-100 (cat. no. ZA0507; 1:100; Beijing Zhongshan Golden Bridge Biotechnology Co., Ltd.), monoclonal rabbit anti-human vimentin (cat. no. M072529; 1:50; Dako) and monoclonal mouse anti-human CEA (cat. no. ZA0507; 1:100; Beijing Zhongshan Golden Bridge Biotechnology Co., Ltd.) antibodies at $4^{\circ} \mathrm{C}$ for $12 \mathrm{~h}$ and visualized under a microscope (CX31; Olympus Corporation). Immunohistochemical examination (Fig. 1A and B) revealed that the tumor was positive for S-100, $\mathrm{CgA}$, Syn, vimentin and CEA expression. The patient recovered well following the procedure and was discharged 11 days post-surgery.

Case 2. A 44-year-old man, who had experienced continuous upper abdominal pain for 6 months was admitted to the People's Hospital of Jiangyou in March 2012. The patient demonstrated no notable abnormalities on physical examination and serum biochemical tests. In addition, the patient did not have a history of hypertension or a remarkable family history. CT scanning revealed a soft-tissue lesion located in the pancreatic uncinate process, measuring $3.2 \times 3 \mathrm{~cm}$, together with a normal pancreatic duct and common bile duct. The presence of the lesion was confirmed by magnetic resonance imaging (MRI; GE 1.5T Signa HDe; GE Healthcare Life Sciences). Due to concerns over the presence of an undetected malignancy, a diagnostic laparotomy was performed, which confirmed that the mass was located in the uncinate process of the pancreas without distorting the surrounding tissues. During surgery, fast-frozen pathological testing of the resected mass indicated a tumor-like lesion, which was suspected to be a high-grade pancreatic neuroendocrine tumor or cystic adenoma. A histological diagnosis of pancreatic pheochromocytoma was confirmed by pathologists following successful local resection of the pancreatic tumor. The tumor cells of the resected tissue were positive for S-100, Syn, CgA and inhibin expression (Fig. 1C). The patient experienced the post-operative complication of a pancreatic fistula, which was treated with drug therapy $(0.1 \mathrm{mg}$ sandostatin subcutaneously injected 3 times/day for 7 days) and percutaneous drainage, which prolonged the duration of the patient's hospital stay. The patient was subsequently discharged almost 2 months after surgery.

Case 3. A 30-year-old woman was admitted the West China Hospital of Sichuan University (Chengdu, China) in August 2011 due to the positive identification of a neoplasm in the pancreatic tail during routine abdominal US. The patient did not exhibit any typical symptoms associated with this disease, and had no history of hypertension. A physical examination and tumor-associated marker evaluation revealed no abnormalities. Following admission to hospital, abdominal enhanced CT detected a mass, $\sim 6 \mathrm{~cm}$ in size, situated in the tail of the pancreas (Fig. 1D). Considering the rapid growth of the tumor and its potential malignancy, a laparotomy was suggested and subsequently performed. However, during surgery, the patient's heart rate and blood pressure suddenly increased to 140 beats/min and $180 / 110 \mathrm{mmHg}$, respectively, as surgeons attempted to dissect the neoplasm. The patient's vital signs were unstable and uncontrollable, which led the surgeons to hypothesize that the tumor may be a pheochromocytoma of the pancreas. Following an emergency consultation, the planned surgery was halted and the patient was transferred immediately to the intensive care unit. The patient's post-surgical blood plasma test revealed noradrenaline and adrenaline levels of 318,306 and $346,940 \mathrm{ng} / \mathrm{l}$, respectively (normal range, $<174,357$ and $<60,104 \mathrm{ng} / \mathrm{l}$, respectively), which confirmed the diagnosis of a pheochromocytoma of the pancreas. Due to an unstable post-operative heart rate and blood pressure, the patient succumbed to heart failure.

\section{Discussion}

Owing to excessive secretion of catecholamines, pheochromocytoma may cause life-threatening symptoms, including hypertension, cardiac arrhythmias, headaches and palpitations (1). However, clinical manifestations of this tumor are variable and non-specific, and in certain cases, the clinical symptoms are not clear. It has been reported that $\sim 70 \%$ of extra-adrenal pheochromocytoma cases are non-functional and only $\sim 30 \%$ of patients may manifest with different presentations; however, the number of patients who do not present with typical symptoms is increasing $(10,11)$.

Due to variable and non-specific manifestations, a diagnosis of extra-adrenal pheochromocytoma is difficult and dependent on clinician experience $(1,2,5)$. In general, a pre-operative diagnosis of extra-adrenal pheochromocytoma is established by taking into account the presence of clinical signs, the determination of catecholamines and their metabolites in blood or urine, and the presence of a mass in the extra-adrenal region. Imaging examinations (US, CT and MRI) are primarily used for locating the lesion, and additionally provide reference values for final histological diagnosis. As previously mentioned, pheochromocytoma derived from the pancreas is seldom observed. Guo et al (9) reported the first pancreatic pheochromocytoma in the Chinese literature: The patient was 40-year-old women that had a mass on the left-upper side of the abdomen for $>1$ month. The patient did not have abdominal pain, distension or discomfort, or a history of trauma, fever, headache, dizziness or high blood pressure. A gigantic pheochromocytoma was identified in the tail of pancreas, and the patient underwent surgery, from which the patient recovered well. The three patients in the present study did not have a history of hypertension nor the typical symptoms associated with this disease. The tumors of the first two patients were located in the uncinate process of the pancreas and were successfully resected. No significant changes in blood pressure or heart rate were detected during surgery, and the diagnosis of a pancreatic pheochromocytoma was primarily based on the pathological and immunohistochemical examination of the resected tissues following surgery. The third patient did not experience any discomfort, but presented with a positive indication of a neoplasm situated in the tail of the pancreas, for which a distal resection was planned as a treatment strategy. However, the surgery was halted intraoperatively due to a sudden increase in the patient's blood pressure and heart rate. Due to a marked post-operative increase in noradrenaline and adrenaline levels in the patient's blood plasma, a diagnosis of pheochromocytoma located in the pancreas was established.

Pheochromocytoma originates in chromaffin cells derived from primitive neural crest cells, which may react and stain 
with chromic salts (8). Furthermore, pheochromocytoma or paraganglioma in the pancreas, to a certain extent, is a type of pancreatic neuroendocrine tumor (pNET), which means that the tumor cells of resected tumors may demonstrate positive reactions for $\mathrm{CgA}$ and Syn following immunohistochemical staining (12). With regard to the first two cases presented here, this pathological feature is of significant value for histological diagnosis of pancreatic pheochromocytoma.

Surgery is the only curative treatment for pNET and pancreatic pheochromocytoma (13). If pheochromocytoma of the pancreas is suspected, surgery should be suggested. Prior to this, pre-operative assessments should be managed for a certain period of time in case of sudden large increases in blood pressure and heart rate. Exploratory laparotomy is a method of diagnosis, but if there are not enough pre-operative preparations, surgery may lead to sudden hypertension resulting in patient mortality, as occurred in the third case reported in the present study. For surgeons who encounter an unexpected hypertensive crisis during pancreatic tumor surgery, an undiagnosed pheochromocytoma of the pancreas should be immediately considered. The surgery should be halted, and appropriate preparations should then be made for a safer second attempt at the surgery.

\section{References}

1. Werbel SS and Ober KP: Pheochromocytoma. Update on diagnosis, localization, and management. Med Clin North Am 79: 131-153, 1995.

2. Sandur S, Dasgupta A, Shapiro JL, Arroliga AC and Mehta AC: Thoracic involvement with pheochromocytoma: A review. Chest 115: 511-521, 1999.
3. Neumann HP, Berger DP, Sigmund G, Blum U, Schmidt D, Parmer RJ, Volk B and Kirste G: Pheochromocytomas, multiple endocrine neoplasia type 2, and von Hipple-Lindau disease. N Engl J Med 329: 1531-1538, 1993.

4. Gimm O, Armanios M, Dziema H, Neumann HP and Eng C: Somatic and occult germ-line mutations in SDHD, a mitochondrial complex II gene, in nonfamilial pheochromocytoma. Cancer Res 60: 6822-6825, 2000

5. Atiyeh BA, Barakat AJ and Abumrad NN: Extra-adrenal pheochromocytoma. J Nephrol 10: 25-29, 1997.

6. Schwartz EL, Mao P, Hernried O, Born EE and Waldmann EB: Catecholamine-secreting paraganglioma. The problem of classification. Arch Intern Med 135: 978-985, 1975.

7. Stackpole RH, Melicow MM and Uson AC: Pheochromocytoma in children. Report of 9 cases and review of the first 100 published cases with follow-up studies. J Pediatr 63: 314-330, 1963.

8. Madani R, Al-Hashmi M, Bliss R and Lennard TW: Ectopic pheochromocytoma: Does the rule of tens apply? World J Surg 31: 849-854, 2007.

9. Guo XY, Wang XJ and Yang JY: A gigantic pheochromocytoma in the tail of the pancreas. He Bei Yi Yao 13: 1628, 2009 (In Chinese).

10. Birrenbach T, Stanga Z, Cottagnoud P and Stucki A: Unexpected metastatic pheochromocytoma - an unusual presentation. Eur J Intern Med 19: 60-62, 2008.

11. Parmer RJ and Zinder O: Catecholaminergic pathways, chromaffin cells, and human disease. Ann NY Acad Sci 971: 497-505, 2002.

12. Panzuto F, Severi C, Cannizzaro R, Falconi M, Angeletti S, Pasquali A, Corleto VD, Annibale B, Buonadonna A, Pederzoli P and Delle Fave G: Utility of combined use of plasma levels of chromogranin A and pancreatic polypeptide in the diagnosis of gastrointestinal and pancreatic endocrine tumors. J Endocrinol Invest 27: 6-11, 2004.

13. Halfdanarson TR, Rabe KG, Rubin J and Petersen GM: Pancreatic neuroendocrine tumors (PNETs): Incidence, prognosis and recent trend toward improved survival. Ann Oncol 19: 1727-1733, 2008. 